\title{
Modulation of microRNA activity by semi-microRNAs
}

\author{
Isabelle Plante ${ }^{1,2}$, Hélène Plé ${ }^{1,2}$, Patricia Landry ${ }^{1,2}$, Preethi H. Gunaratne ${ }^{3,4,5}$ and Patrick Provost ${ }^{1,2 *}$ \\ ${ }^{1}$ CHUQ Research Center/CHUL, Quebec, QC, Canada \\ 2 Faculty of Medicine, Université Laval, Quebec, OC, Canada \\ ${ }^{3}$ Department of Biology and Biochemistry, University of Houston, Houston, TX, USA \\ ${ }^{4}$ Department of Pathology, Baylor College of Medicine, Houston, TX, USA \\ ${ }^{5}$ Human Genome Sequencing Center, Baylor College of Medicine, Houston, TX, USA
}

\section{Edited by:}

Muller Fabbri, Childrens' Hospital Los Angeles and Keck School of Medicine University of Southern California, CA, USA

\section{Reviewed by:}

Francesca Fanini, Istituto Scientifico Romagnolo per lo Studio e la Cura dei Tumori, Italy

Florian Kuchenbauer, University of Ulm, Germany

*Correspondence:

Patrick Provost, CHUQ Research Center/CHUL, 2705 Blvd Laurier, Room T1-49, Quebec, OC, Canada G1V 4 G2

e-mail: patrick.provost@

crchul.ulaval.ca
The ribonuclease Dicer plays a central role in the microRNA pathway by catalyzing the formation of 19-24-nucleotide (nt) long microRNAs. Subsequently incorporated into Argonaute 2 (Ago2) effector complexes, microRNAs are known to regulate messenger RNA (mRNA) translation. Whether shorter RNA species derived from microRNAs exist and play a role in $m R N A$ regulation remains unknown. Here, we report the serendipitous discovery of a 12-nt long RNA species corresponding to the $5^{\prime}$ region of the microRNA let-7, and tentatively termed semi-microRNA, or smiRNA. Using a smiRNA derived from the precursor of miR-223 as a model, we show that 12-nt long smiRNA species are devoid of any direct mRNA regulatory activity, as assessed in a reporter gene activity assay in transfected cultured human cells. However, smiR-223 was found to modulate the ability of the microRNA from which it derives to mediate translational repression or cleavage of reporter mRNAs. Our findings suggest that the 12-nt RNA species, generated along the microRNA pathway, may participate to the control of gene expression by regulating the activity of the related full-length mature microRNA in vivo.

Keywords: microRNA, non-coding RNA, Dicer, Argonaute 2, gene regulation, RNA silencing

\section{INTRODUCTION}

Small non-coding RNAs, such as microRNAs, have recently evolved as key players in the fine tuning of gene expression, which is required for preserving well balanced protein levels and maintaining normal cellular functions. As short as $19-24 \mathrm{nt}$ in length, microRNAs may regulate $\sim 60 \%$ of the genes (Bartel, 2009), suggesting that potentially every metabolic process may be under microRNA control in our body. In humans, the biogenesis of microRNAs is mediated by the ribonuclease (RNase) Dicer (Provost et al., 2002; Zhang et al., 2002), a large multidomain protein that we originally identified as a protein interacting with 5-lipoxygenase (5LO; Provost et al., 1999). Dicer forms a complex with transactivating response (TAR) RNA binding protein (TRBP; Chendrimada et al., 2005; Haase et al., 2005) and processes microRNA precursors (pre-microRNAs), through the concerted action of its C-terminal RNase IIIa and IIIb motifs, to generate a microRNA duplex composed of a $5 p$ and $3 p$ microRNA strand. Recent studies have shown that either strand may subsist and nucleate the formation of Argonaute 2 (Ago2) silencing complexes competent in the regulation of specific messenger RNAs (mRNAs; Okamura et al., 2008; Guo and Lu, 2010; Mah et al., 2010; Yang et al., 2011).

The ability of microRNAs to inhibit translation or induce degradation of target mRNAs is closely correlated to the degree of microRNA:mRNA complementarity (Bartel, 2009). Acting mainly through base pairing of their seed region (Bartel, 2009), microRNAs initially repress mRNA translation through recognition of specific binding sites (BS) located mainly, but not exclusively, in the $3^{\prime}$ untranslated region ( $3^{\prime}$ UTR) of mRNAs, as targeting may also occur in the 5'UTR (Lytle et al., 2007) and open-reading frame (Lewis et al., 2005; Qin et al., 2010). MicroRNAs have also been shown to activate the expression of a target gene (Vasudevan et al., 2007).

Whether shorter RNA species derived from microRNAs exist and play a role in mRNA regulation remains unknown. While using a high-throughput sequencing (HTS) approach aimed to elucidate the small RNA profile of human platelets, which contain an abundant and diverse array of microRNAs (Landry et al., 2009), we serendipitously discovered a significant number of 12-nt long RNA sequences. Corresponding to the $5^{\prime}$ half of the microRNA let-7, we tentatively termed these 12 -nt RNA species semi-microRNA, or smiRNA. Devoid of intrinsic, direct mRNA regulatory activity, a 12-nt model smiRNA was found to modulate the ability of the microRNA from which it derives to mediate translational repression or cleavage of reporter mRNAs. Our findings suggest that smiRNAs may represent a novel class of small non-coding RNAs generated along the microRNA pathway and capable of regulating microRNA activity in vivo.

\section{MATERIALS AND METHODS HUMAN PLATELET PURIFICATION AND RNA EXTRACTION}

Human platelets were isolated from venous blood collected from healthy volunteers, as described previously (Landry et al., 2009). Purified platelets were harvested by centrifugation at $6,000 \mathrm{~g}$ for $1 \mathrm{~min}$, and total RNA was extracted using TRIzol reagent (Invitrogen). Equal amounts of platelet total RNA, extracted from five different subjects, were pooled together, and purified further using 
an RNeasy Mini kit (Qiagen). The integrity of platelet RNA was assessed using Bioanalyzer 2100 (Agilent) prior to further analyses.

\section{SMALL RNA LIBRARY CONSTRUCTION AND SEQUENCING}

A small RNA library was constructed from $15 \mu \mathrm{g}$ of total RNA, extracted from five different subjects, by isolating RNA species between 18 and $30 \mathrm{nt}$ in length using the DGE small RNA sample prep kit (Illumina), following the manufacturer's instructions. The labeled cDNAs were quantified with the Quant-iT PicoGreen dsDNA Kit (Invitrogen) and diluted to $3 \mathrm{pM}$ for sequencing in a single lane on an Illumina GA-I and GA-II Genome Analyzers (Solexa), essentially as described previously (Creighton et al., 2010).

Raw sequences were filtered through serial quality control criteria. First, the presence of at least $6 \mathrm{nt}$ of the $3^{\prime}$ Solexa adapter was verified. The sequence reads that did not comply with this criterion were discarded, whereas the others were trimmed to remove the adapter sequence harbored at the $3^{\prime}$ end. The remaining tags were further filtered regarding their length ( $>10 \mathrm{nt})$, copy number $(>4$ reads), and readability $(<9 \mathrm{~N})$. Reads complying with all those criteria were subsequently defined as usable reads.

All the usable reads were aligned to pre-microRNAs extracted from miRBase database (release 18.0). Sequence tags that matched perfectly to more than one precursor were distributed equally among them. The relative abundance of each microRNA species was defined as the number of reads mapping to the corresponding pre-microRNA compared to the total number of reads mapping the mature microRNA species.

\section{CELL LINE AND CULTURE}

Cultured human HEK 293 cells (obtained from ATCC) were grown in Dulbecco's modified Eagle's medium (DMEM) supplemented with $10 \%(\mathrm{v} / \mathrm{v})$ fetal bovine serum (FBS), $1 \mathrm{mM}$ sodium pyruvate, 100 units/mL penicillin, $100 \mu \mathrm{g} / \mathrm{mL}$ streptomycin, and $2 \mathrm{mM}$ L-glutamine in a humidified incubator under $5 \% \mathrm{CO}_{2}$ at $37^{\circ} \mathrm{C}$.

\section{REPORTER GENE CONSTRUCTS}

Several psiCHECK-based, Dual Luciferase reporter gene constructs, in which well-defined regulatory elements have been introduced at the $3^{\prime}$ end of the Renilla luciferase (Rluc) open-reading frame (Boissonneault et al., 2008), have been designed in order to study the regulatory role of smiRNAs in mRNA translation and/or microRNA activity. The target sequences were either amplified by PCR or obtained by annealing oligonucleotides, and cloned downstream of the Rluc reporter gene in the XhoI/NotI sites of the psiCHECK (Promega) reporter vector.

\section{REPORTER GENE ACTIVITY ASSAY}

HEK 293 cells were co-transfected by the calcium phosphate method, at $\sim 50 \%$ confluence in 24 -well plates, with a synthetic smiR-223 alone $(25-250 \mathrm{pmol})$, or with a synthetic smiR-223 (25-50 pmol) and a psiSTRIKE (Promega) vector expressing the precursor of miR-223 (2.5 or $25 \mathrm{ng}$ ), and a psiCHECK reporter construct (50 ng; Promega) in which the Rluc reporter gene is coupled with a BS of perfect complementarity (PC) to miR-223 or to three copies of a natural BS to miR-223, either wild-type (WT) or mutated (MUT) to disrupt base pairing to the miR-223 seed region. For that latter purpose, we selected the natural BS to miR223 that is present in the $3^{\prime} \mathrm{UTR}$ of humanP2 $\mathrm{Y}_{12}$ mRNA and shown to be regulated by platelet miR-223 (Landry et al., 2009). Cells were harvested $48 \mathrm{~h}$ later, and Rluc and Firefly luciferase (Fluc) activities were measured, as described previously (Landry et al., 2009). Results of Rluc activity were normalized with Fluc reporter activity and expressed as a percentage of the results obtained with an unrelated smiRNA and/or a construct expressing an unrelated pre-microRNA (set at 100\%).

\section{5' PHOSPHORYLATION STATUS OF SMALL RNAs}

Total RNA was extracted from cultured HEK 293 cells (left panel) or primary human platelets (right panel) with Trizol, and enriched in small RNAs $(<200 \mathrm{nt})$ by using the mirVana small RNA isolation kit (Ambion). The small RNA preparations (10,20, or $160 \mathrm{ng}$ ) were either dephosphorylated with calf intestine phosphatase (Roche) or left untreated, and $5^{\prime}$ end labeled with ${ }^{32}$ P-ATP (Perkin Elmer) using T4 polynucleotide kinase (Affymetrix). ${ }^{32} \mathrm{P}$-labeled ATP incorporation in small RNAs was analyzed by denaturing polyacrylamide gel electrophoresis (PAGE) and autoradiography.

\section{RESULTS}

\section{DETECTION OF A 12-nt LONG RNA SEQUENCE DERIVED FROM hsa-let-7 MICRORNA}

During the course of an HTS experiment of human platelet small RNAs, we serendipitously discovered a significant number of 12-nt long RNA sequences corresponding to the $5^{\prime}$ half of the microRNA let-7 and tentatively termed semi-microRNA, or smiRNA. The fortuitous discovery of such a small RNA species may emanate from a slightly imprecise sampling of the gel during the size-fractionation step aimed to isolate (and analyze) RNA species in the standard 1830-nt range. The 12-nt long sequence 5'-UGAGGUAGUAGG-3' was the fifth most abundant RNA species mapping to the hsalet-7c pre-microRNA, with 63 copies. Interestingly, this sequence mapped to the region corresponding to the $5^{\prime}$ half, seed-containing mature let-7 (Figure 1A). The formation of let-7 smiRNAs may be initiated either by (i) sequential processing events involving Drosha and Dicer, followed by further processing of the microRNA strand in half (Figure 1B), or (ii) direct processing of the $5^{\prime}$ end of the pre-microRNA species.

Further analyses of our data also revealed the existence of smiRNAs derived from miR-223. For instance, the RNA species $5^{\prime}$-UGUCAGUUUGUCAAA- $3^{\prime}$ was found in 139 copies in primary human neutrophils (Figure 1C), thereby extending our let-7c smiRNA findings to miR-223 and rationalizing the use of smiRNA-223 for subsequent functional studies. It is interesting to note that this short RNA species is absent in platelets, where miR-223 is abundant and functional.

\section{SmiRNAs MAY NOT DIRECTLY MEDIATE GENE SILENCING THROUGH mRNA CLEAVAGE}

In order to assess the functionality of these unusually short RNA species, we have performed Dual-Luciferase reporter gene assays in transiently transfected HEK 293 cells (Plante et al., 2006; Ouellet et al., 2008; Boissonneault et al., 2009; Landry et al., 2009). We have studied two different types of microRNA regulatory elements, which were inserted in the 3'UTR of the Rluc reporter 


\section{A}

hsa-let-7c (hsa-let-7c)

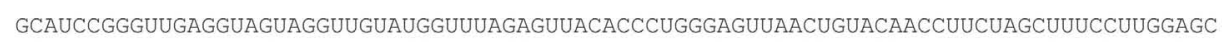

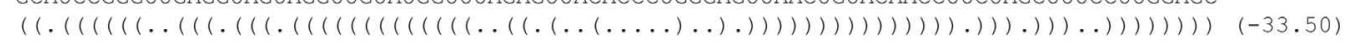

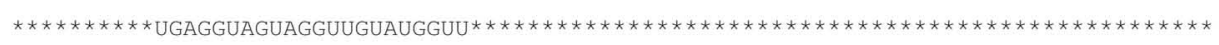

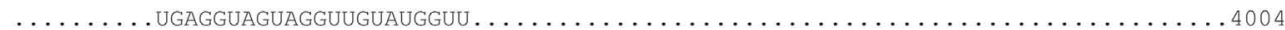

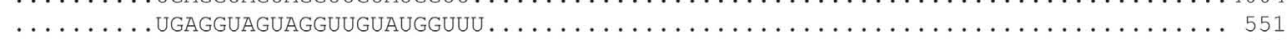

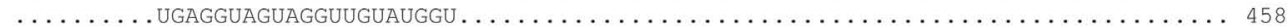

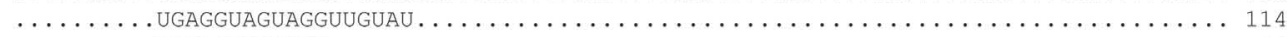

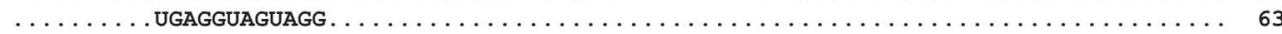

\section{B}

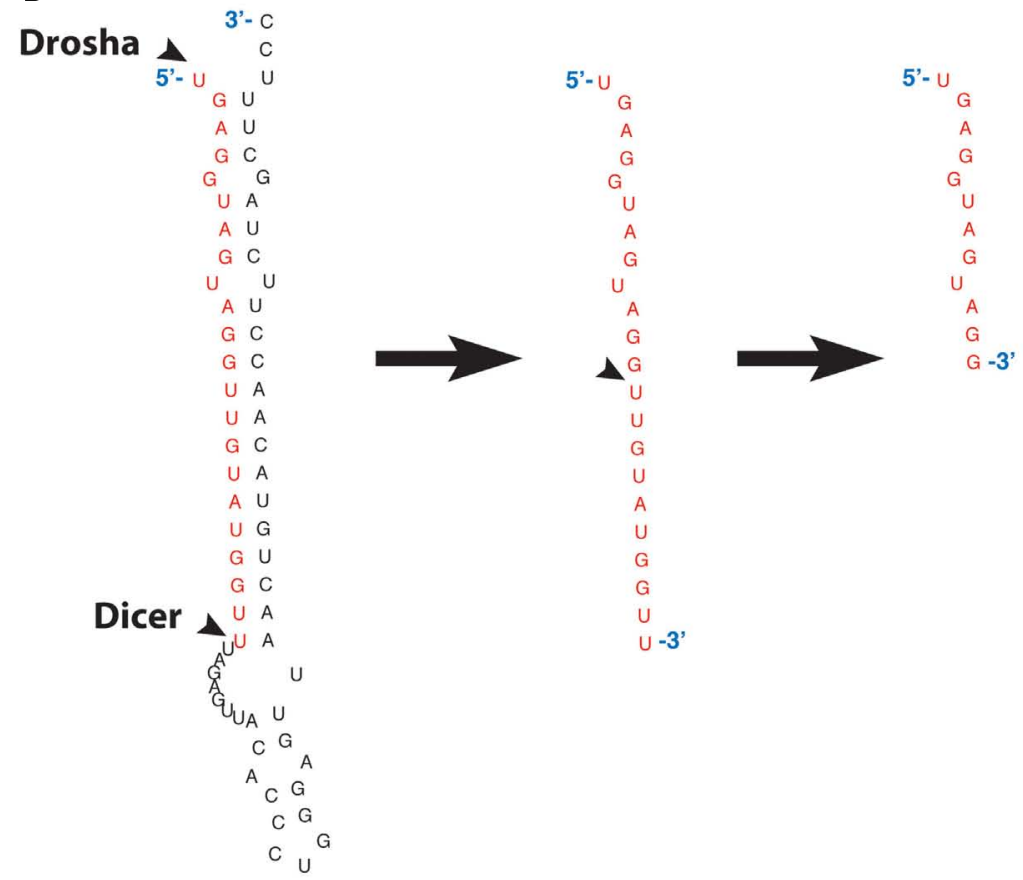

hsa-pre-let-7c

C 12-nt hsa-let-7

(smiRNA)

hsa-mir-223 (hsa-mir-223)

CCUGGCCUCCUGCAgUgCCACGCUCCGUGUAUUUGACAAGCUGAGUUGGACACUCCAUGUGGUAGAgUguCAGUUUGUCAAAUACCCCAAGUGCGGCACAUGCUUACCAG

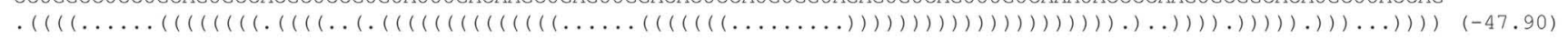

$* * * * * * * * * * * * * * * * * * * * * * * * * * * * * * * * * * * * * * * * * * * * * * * * * * * * * * * * * * * * * * * * * * *$ UGUCAGUUUGUCAAAUACCCCA $* * * * * * * * * * * * * * * * * * * * *$

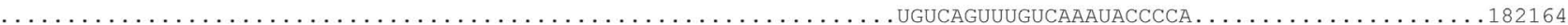

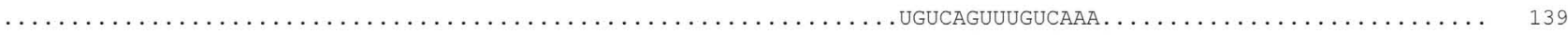

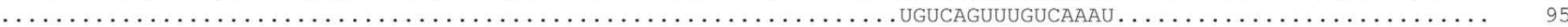
.

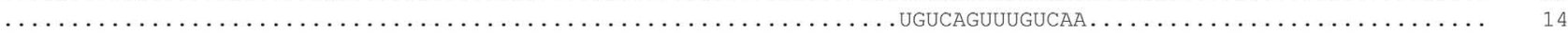

FIGURE 1 | Detection of a 12-nt long RNA sequence derived from hsa-let-7 microRNA. (A) Sequences obtained upon analysis of primary human platelet small RNAs by high-throughput sequencing (HTS) were aligned to the human genome and mapped to microRNA genes (miRBase Release 18). The five most abundant RNA species mapping to the hsa-let-7c pre-microRNA are shown. More than 4004 sequences reads represented mature let-7c microRNA, whereas 63 sequences derived from the $5^{\prime}$ half of mature let-7c microRNA. (B) Secondary structure of the hsa-pre-let-7c, as predicted by MFOLD. The arrowheads indicate the
Drosha and Dicer cleavage sites that yield mature hsa-let-7c microRNA, which may be further processed into a 12-nt hsa-let-7c RNA species half the size of the mature hsa-let-7c microRNA. Tentatively termed semi-microRNA (or smiRNA), these 12-nt hsa-let-7c RNAs may be generated upon direct processing of the $5^{\prime}$ end of the pre-microRNA species. (C) Sequences obtained upon analysis of primary human neutrophil small RNAs by HTS, as in A. RNA species of a length (14-16 nt) similar to smiRNAs, mapping to the hsa-miR-223 pre-microRNA, are shown. 
gene (Fluc serving as a normalization control): (i) a synthetic BS of PC, through which microRNAs mediate mRNA cleavage, and (ii) three copies of a natural WT BS of imperfect complementarity, through which microRNAs mediate mRNA translational repression. Inactivated versions of the natural WT BS have been created by mutating the microRNA seed region (MUT), whose pairing is critical to mediate microRNA function (Bartel, 2009).

To study the gene regulatory effects of smiRNAs, we have used a model smiRNA designed on hsa-miR-223, an abundant and functional platelet microRNA known to recognize a BS located in the 3'UTR of human P2Y 12 mRNA (Landry et al., 2009). miR-223 was selected as the model smiRNA over let-7 miRNA, since the latter is present in several isoforms and expressed at various extent in human cell lines, in contrast to miR-223, whose expression is more restricted to cell types of the hematopoiesis lineage. The fact that let-7 microRNAs share the same seed region would have generated a non-negligible background signal that would have hampered the detection of a positive effect and complicated the interpretation of reporter gene activity data obtained from the use and study of a let-7 smiRNA.

To test whether smiR-223 can exert direct mRNA regulatory effects, we co-transfected synthetic smiR-223 with an Rluc reporter gene harboring a target sequence perfectly complementary to smiR-223 (Figure 2A). Rluc reporter mRNA cleavage and a corresponding decrease in Rluc activity would be expected for a smiR-223 functional in mRNA regulation. However, smiRNAs derived either from the $5^{\prime}$ or the $3^{\prime}$ half of hsa-miR-223 did not exert any reporter gene regulatory effects (Figure 2B).

\section{SMIRNAs MAY NOT DIRECTLY MEDIATE GENE SILENCING THROUGH mRNA TRANSLATIONAL REPRESSION}

To determine whether smiR-223 can mimic miR-223 function and repress mRNA translation, we designed Rluc reporter gene constructs in which three copies of the natural (miR-223 $3 \times \mathrm{BS}$ WT; dark blue) or MUT (miR-223 $3 \times$ BS MUT; light blue) BS to miR-223 was placed downstream of Rluc (Figure $\mathbf{3 A}$ ). This approach aimed to more closely mimic the situation in human cells (Boissonneault et al., 2008), where microRNAs act in concert to coordinately regulate mRNA translation through imperfect base pairing. In these experiments, smiR-223 also failed to induce any direct gene regulatory effects (Figure 3B), suggesting that 12-nt smiRNA species may not conceal any intrinsic mRNA regulatory activity, whether the regulatory elements are of $\mathrm{PC}$ or not.

\section{smiRNAs MAY REGULATE THE GENE SILENCING PROPERTIES OF MICRORNAs}

We then examined whether smiRNAs could interfere with microRNA function. Expression of pre-miR-223 induced a marked decrease in the activity of anRluc reporter gene placed under the control of a BS of PC to miR-223 (Figure 4A). Cotransfection of synthetic smiR-223 inhibited the mRNA regulatory function of miR-223 and restored Rluc expression to

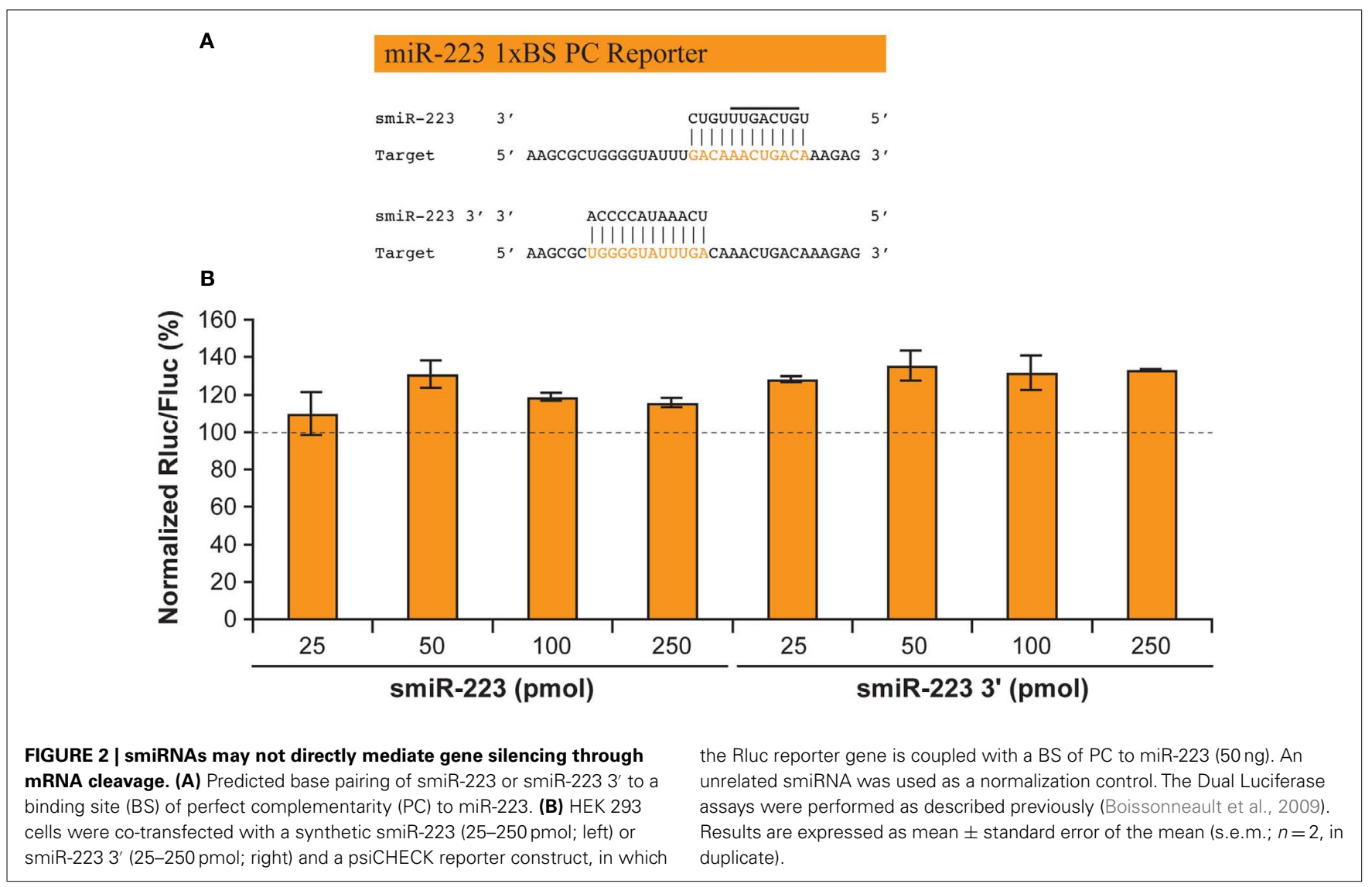




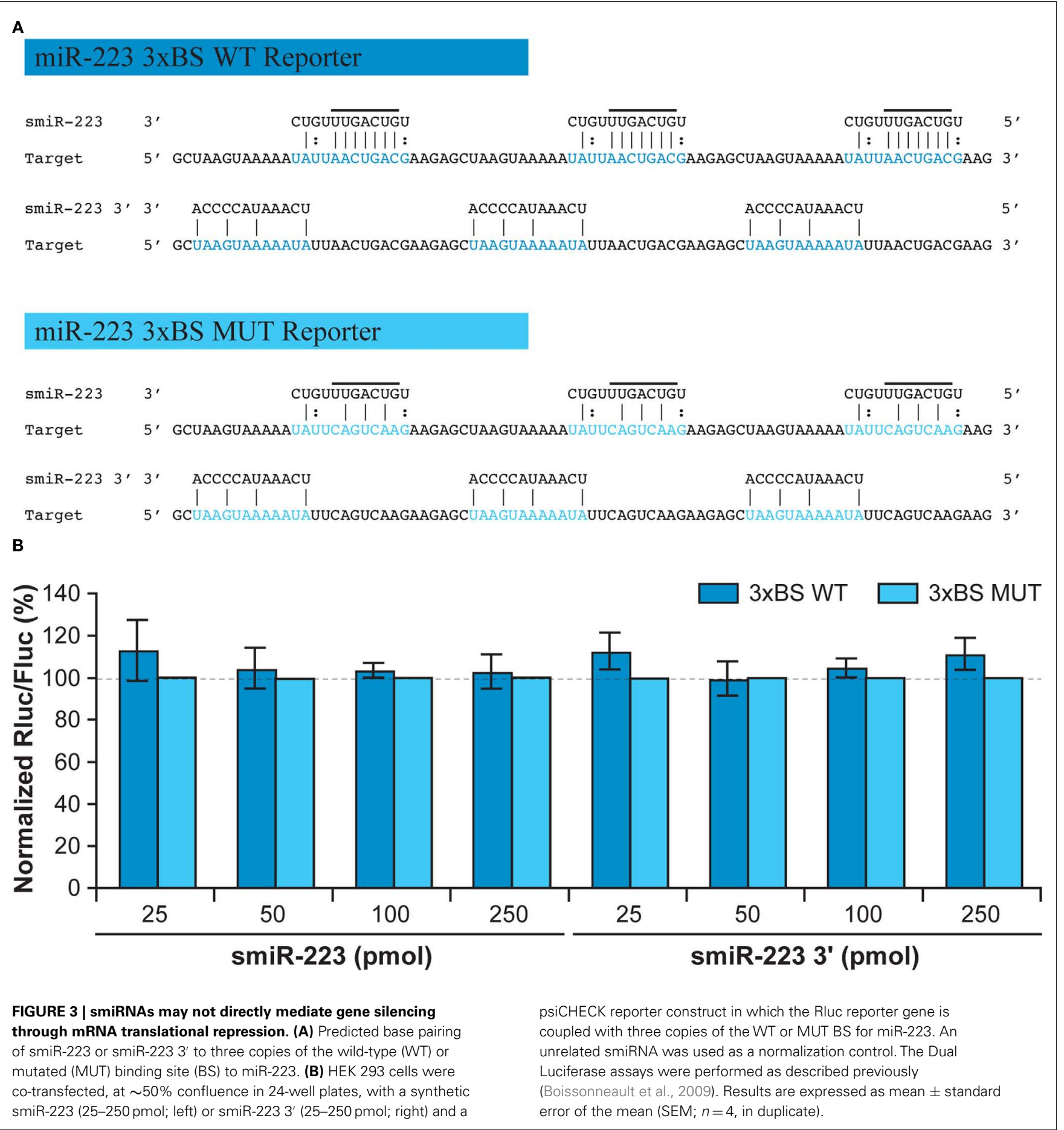

normal levels (Figure 4A). Similar results were obtained in reporter gene experiments performed in the context of microRNAinduced mRNA translational repression, in which smiR-223 dosedependently prevented the mRNA regulatory activity of miR-223 (Figure 4B). These results suggest that smiR-223 can interfere with the ability of miR-223 to mediate gene silencing in human cells, either through mRNA cleavage or mRNA translational repression.

\section{5' PHOSPHORYLATION STATUS OF SMALL RNAs}

These differential effects of smiRNAs prompted us to investigate the $5^{\prime}$ phosphorylation status of smiRNAs. Considering (i) that $5^{\prime}$ end phosphorylation is required for small RNA anchorage in the G1 binding pocket of Ago2 (Ma et al., 2005) and function in mRNA silencing, and (ii) the lack of any direct mRNA regulatory effect of smiRNAs, we suspected that the $5^{\prime}$ end of $\sim 12$-nt smiRNA species may not be phosphorylated. 


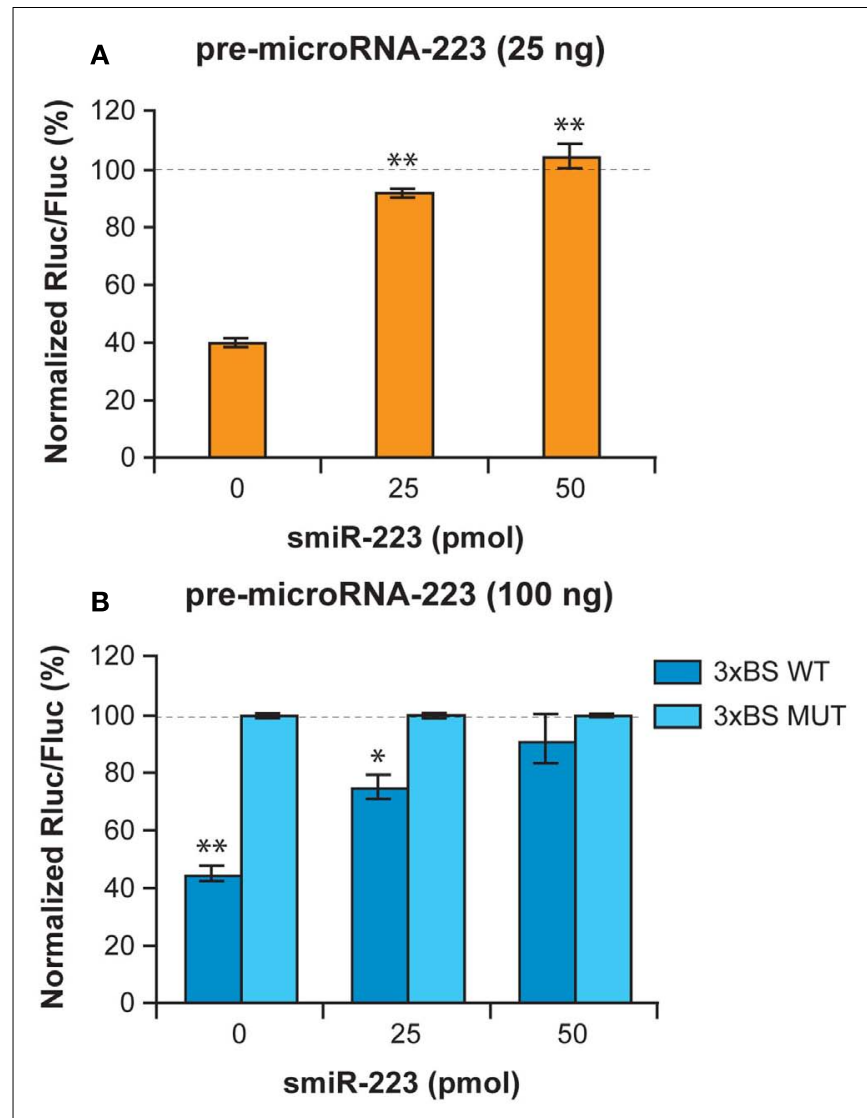

FIGURE 4 | smiRNAs may regulate the gene silencing properties of microRNAs. (A,B) HEK 293 cells were co-transfected with a synthetic smiR-223 (0, 25, or 50 pmol), a psiSTRIKE vector expressing pre-miR-223 ( 2.5 or $25 \mathrm{ng}$ ) and a psiCHECK reporter construct $(50 \mathrm{ng})$, in which the Rluc reporter gene is coupled either (A) to a binding site (BS) of perfect complementarity (PC) to miR-223, or (B) three copies of the wild-type (WT) or mutated (MUT) BS for miR-223. An unrelated smiRNA and a construct expressing an unrelated pre-microRNA were used as normalization controls. The Dual Luciferase assays were performed as described previously (Boissonneault et al., 2009). Results are expressed as mean \pm standard error of the mean (s.e.m.; $n=2-4$, in duplicate). ${ }^{*} p<0.05$ or ${ }^{* *} p<0.01$ versus $0 \mathrm{pmol}$ smiR-223 (A) or versus the $3 \times \mathrm{BS}$ MUT (B) (Student's $t$ test).

To verify that possibility, we subjected small RNAs ( $<200 \mathrm{nt})$ extracted from cultured HEK 293 cells or primary human platelets, dephosphorylated or left untreated, to $5^{\prime}$ end labeling with ${ }^{32} \mathrm{P}$-ATP using T4 polynucleotide kinase. In these experiments, endogenous 12-nt smiRNA species phosphorylated at their $5^{\prime}$ end will be phosphorylated in vitro (and visualized) only if they are dephosphorylated first. Analysis of ${ }^{32} \mathrm{P}$-labeled ATP incorporation by denaturing PAGE and autoradiography revealed the presence of phosphorylable RNA species of 10 and $12 \mathrm{nt}$ in length, predominantly in HEK 293 cells (Figure 5, lanes 1 and 4). However, an important proportion, if not most, RNA species in the range of sizes expected for smiRNAs are not phosphorylated at their $5^{\prime}$ end in vivo (Figure 5, lanes 2 and 5). This may explain, at least in part, why smiRNAs are devoid of intrinsic, direct mRNA regulatory activity.

\section{DISCUSSION}

In this study, we report the identification of 12-nt RNA species half the size of microRNAs, that we tentatively termed semimicroRNAs (or smiRNAs). Devoid of intrinsic, direct mRNA regulatory activity, a 12-nt model smiRNA was found to modulate the ability of the microRNA from which it derives to mediate translational repression or cleavage of reporter mRNAs. Our findings suggest that smiRNAs may represent a novel class of small non-coding RNAs generated along the microRNA pathway and capable of regulating microRNA activity in vivo.

The 12-nt sequence detected in our HTS experiment (UGAGGUAGUAGG - 63 reads) is common to, and likely derive from, let-7a (468,491 reads), let-7b (87,483 reads), and let-7c (5305 reads). Discovered serendipitously, the relatively low abundance of this 12-nt smiRNA sequence, as compared to the corresponding full-length mature microRNA, may be related to (i) its inherently low and/or transient expression level, (ii) its relative instability, or (iii) a technically imprecise size fractionation, as the length of the RNA species to be analyzed by HTS was initially set to a standard 18- to 30-nt window. As opposed to a smear of sequences of various lengths, the significant number of well-defined, discrete RNA sequences of $12 \mathrm{nt}$ in length and derived from microRNAs argues against smiRNAs being non-specific degradation products.

Twelve-nucleotides long RNA species may represent byproducts of processing events involving Ago2. It is worth noting that Matranga et al. (2005) detected the presence of a 9-nt RNA species that accumulated early, but disappeared within $30 \mathrm{~min}$, upon passenger-strand cleavage of a 21 -nt siRNA, along a reaction that may also have liberated a 12-nt RNA by-product. However, although Ago2-mediated passenger-strand cleavage of doublestranded siRNAs is required for RISC assembly, it does not appear to be important for the incorporation of microRNAs that derive from mismatched duplexes (Matranga et al., 2005), such as those found in let-7 pre-microRNAs. Let-7b pre-microRNA may be an exception, as the let-7b-5p:let-7b-3p microRNA duplex is perfectly base-paired, except for nt 1 of let-7b-5p (Figure A1 in Appendix).

Twelve-nucleotides long RNAs may also originate from Ago2 processing of pre-microRNA species, as demonstrated by Diederichs and Haber (2007). They observed that Ago2cleaved let-7a-3 precursor microRNAs, cloned from 293 cells cotransfected with let-7a-3 and Ago2, were shortened by $\sim 12$ nt at their $3^{\prime}$ end. Of note, the abundance of the ac-pre-miRNA derived from non-transfected cells expressing endogenous microRNA and Ago2 protein was lower than that observed in cells expressing ectopic microRNA and Ago2 protein, thereby explaining why the endogenous precursors were not readily detectable by Northern blotting (Diederichs and Haber, 2007). This endogenous, on-pathway intermediate, resulting from cleavage of the premicroRNA hairpin $12 \mathrm{nt}$ from its $3^{\prime}$ end, may thus represent an endogenous source of RNAs that are 12-nt in length. However, since the mechanism leading to their formation involves cleavage of the $3^{\prime} \mathrm{arm}$, this would not explain the formation of the 12-nt smiRNA sequence that we detected, which is derived from the $5^{\prime}$ arm of pre-let-7.

Interestingly, ac-pre-miRNAs appeared to be processed as efficiently as pre-microRNAs by Dicer in vitro (Diederichs and Haber, 


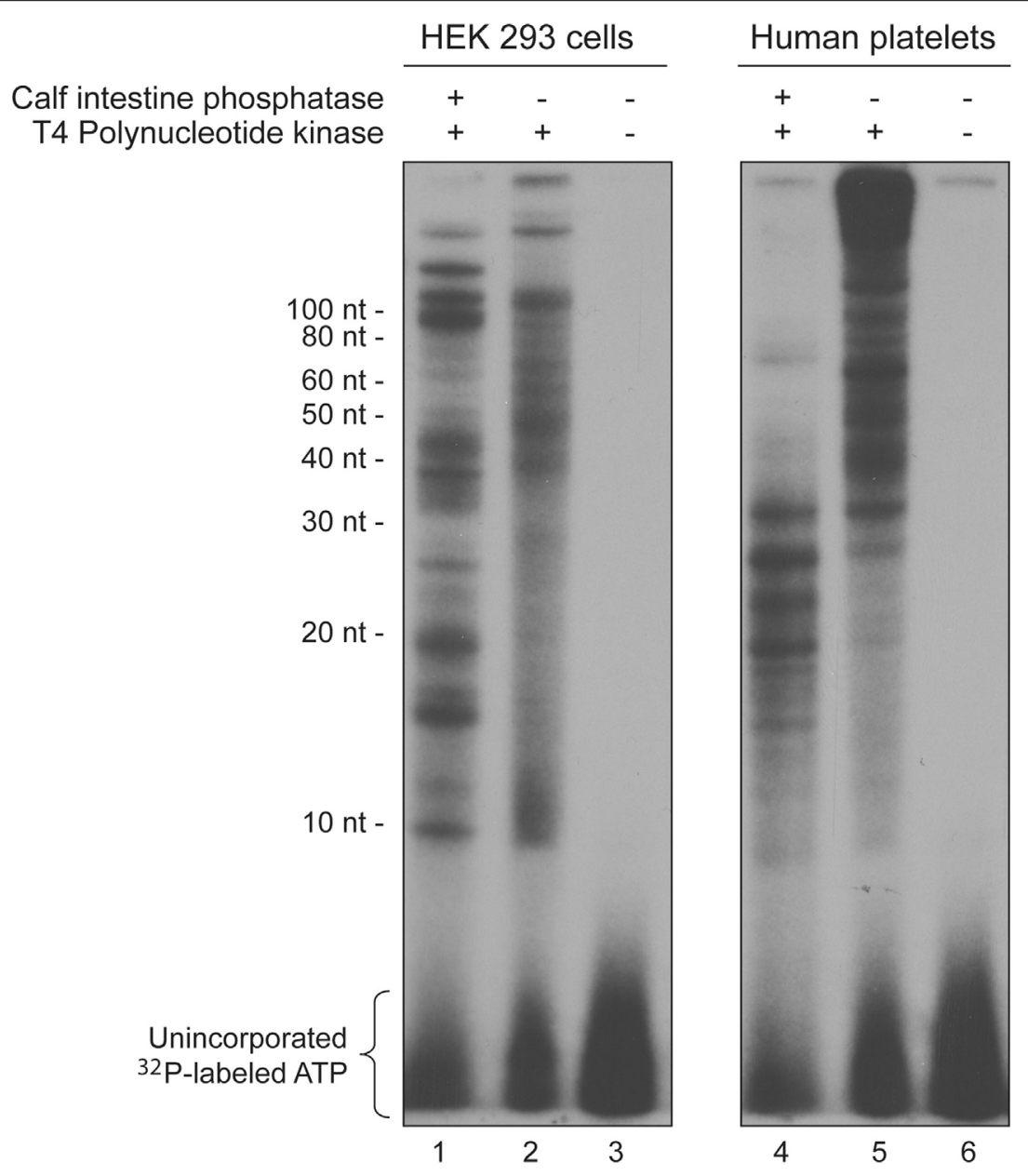

FIGURE 5 | 5' Phosphorylation status of small RNAs. Small RNAs (<200 nt) extracted from cultured HEK 293 cells (left panel, lanes 1-3) or primary human platelets (right panel, lanes 4-6) were either dephosphorylated with calf intestine phosphatase (+; lanes 1 and 4), or not (-; lanes 2, 3, 5, and 6), and 5' end labeled with ${ }^{32}$ P-ATP using T4 polynucleotide kinase (lanes 1, 2, 4, and 5). ${ }^{32}$ P-labeled ATP incorporation at the $5^{\prime}$ end of small RNAs was analyzed by denaturing PAGE and autoradiography.
2007), suggesting that $\sim 12$-nt species may derive from the sequential processing of pre-microRNAs by Ago2 and Dicer. Recent evidences from our laboratory indicate that Dicer may also contribute to the formation of 12-nt smiRNAs independently of Ago2. Indeed, we observed that the Dicer-interacting protein 5LO, an enzyme expressed mainly in differentiated inflammatory cells but absent from bacteria, could modify the enzymatic properties of the Dicer and induce the formation of RNA species that are $\sim 10-12 \mathrm{nt}$ in length from a pre-microRNA substrate in vitro (Dincbas-Renqvist et al., 2009). This activity is concealed in the C-terminal catalytic domain of human Dicer (a.a. 1650-1912; Dincbas-Renqvist et al., 2009), which may sustain conformational changes upon binding of 5LO. This is supported by the ability of 5LO to interact with the double-stranded RNA (dsRNA) binding domain (dsRBD) of human Dicer (Dincbas-Renqvist et al., 2009). The function of Dicer dsRBD remains unclear, considering that (i) the C-terminal dsRBD is dispensable for processing activity in E. coli RNase III (Sun et al., 2001), (ii) Giardia Dicer lacks a dsRBD domains (Macrae et al., 2006), and (iii) an RNase III naturally lacking the dsRBD functions in B. subtilis (Redko et al., 2008). These observations suggest that human Dicer may have evolved to acquire, through its dsRBD, additional protein interaction functionalities that possibly confer enzymatic properties that are distinct from smaller RNases III. Dicer interaction with a dsRBD-interacting protein, like 5LO, in higher eukaryotes, may thus favor the formation of 12-nt smiRNAs. It will be interesting to test whether 5LO alters the ability of Dicer to form intramolecular dimers, which has been proposed to be required for the generation of RNA products that are twice as long as those derived from bacterial RNases III.

Discovered in primary human platelets, which are devoid of 5LO but expressing a closely related protein (i.e., 12LO), smiRNAs may derive from pre-microRNA species processed by a Dicer complex containing an interacting protein different from 5LO.

Using reporter gene activity assays, we were unable to demonstrate a direct mRNA regulatory role for 12-nt long smiRNA species. The relatively short length of smiRNAs may not hamper their incorporation into Ago2 effector complexes, since Ago2 
can form stable complexes with RNA species as short as $10 \mathrm{nt}$ (Wang et al., 2008). This structural study showed that microRNAs are anchored at their $5^{\prime}$ end, with only the seed region (nt 2-8) being exposed, whereas their $3^{\prime}$ end is buried within the Ago2 structure (Wang et al., 2008). Another important feature of Ago 2 effector complexes is the $5^{\prime}$ phosphorylation of the associated regulatory RNA, which is required for small RNA anchorage in the G1 binding pocket of Ago2 and function in mRNA silencing (Ma et al., 2005). Lacking a phosphate group at their $5^{\prime}$ ends, synthetic siRNAs can be phosphorylated by the kinase hClp1, which allows their assembly into RISC for subsequent target RNA cleavage (Weitzer and Martinez, 2007). The fact that hClp1 can also phosphorylate other nucleic acid molecules - irrespective of length, type of overhang, and whether they are single or doublestranded-raises the question of whether hClp1 has any function in endogenous RNA silencing (Weitzer and Martinez, 2007), including phosphorylation of smiRNAs. However, this latter possibility is not supported by the apparent lack of $5^{\prime}$ phosphorylation of endogenous $\sim 10-12$-nt RNA species, which also argues against the functional incorporation of smiRNAs into Ago 2 complexes. Conferring functional specificities, the unphosphorylated status of smiRNAs may prevent their utilization as guide strands by the Ago2 effector complexes and explain their lack of intrinsic mRNA silencing properties.

Apparently, these findings do not preclude a role for smiRNAs in regulating gene expression, as we were able to demonstrate that smiRNAs can modulate the mRNA silencing activity of the corresponding microRNA from which it derives. Whereas smiRNAs may be long enough to recognize, and compete for, the BS of a microRNA, the relatively low stability, or high free energy

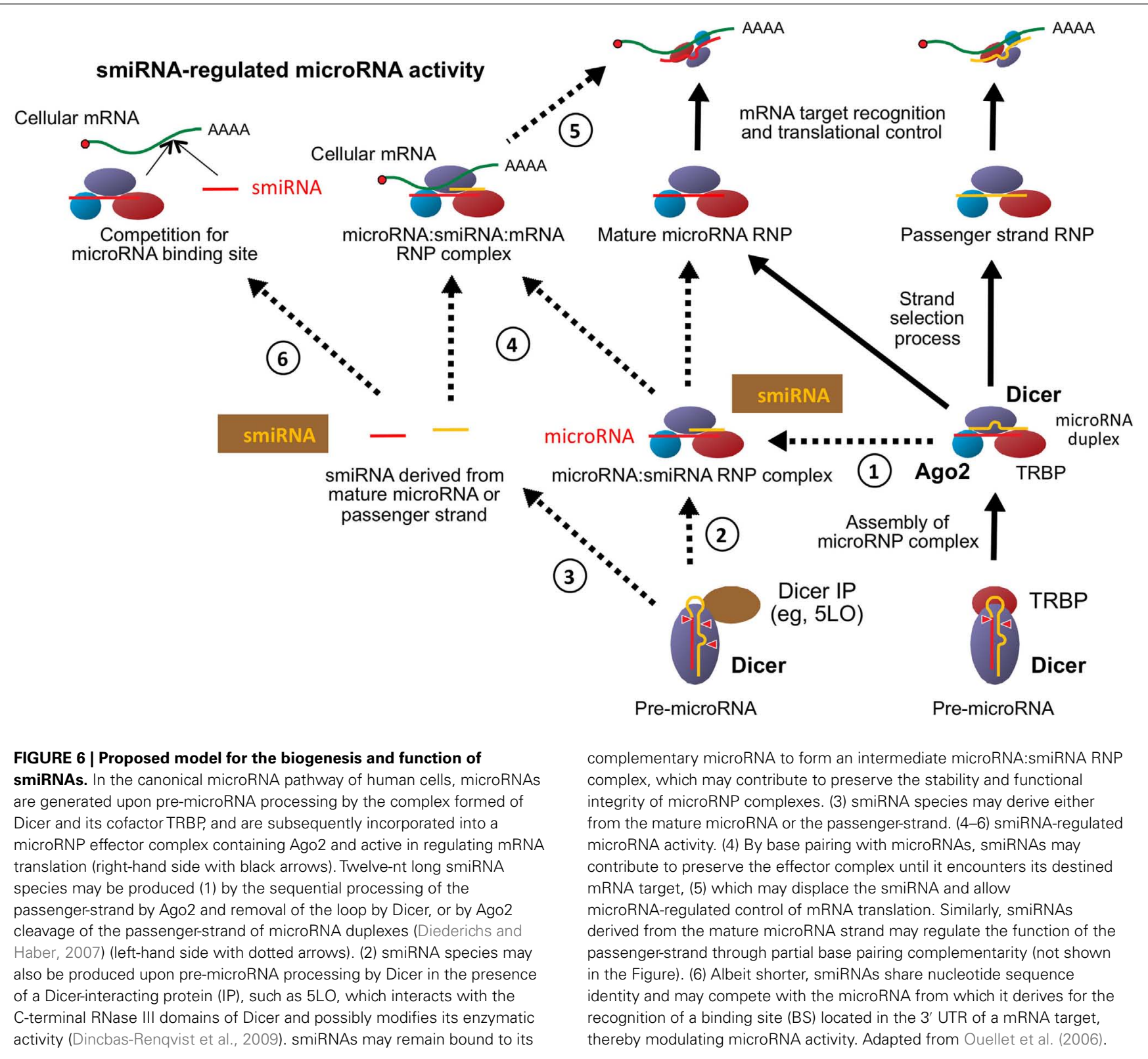


$(\Delta G)$ values, of smiRNA binding to a microRNA BS, combined with the absence of an associated Ago2-containing RNP complex, may make it more susceptible to being dislodged by translocating ribosomes. This scenario would reconcile the lack of direct mRNA regulatory properties of smiRNAs with their ability to regulate microRNA activity. This is in accordance with previous studies reporting (i) that $16 \mathrm{nt}$ is the minimal length required for an RNA to trigger an RNA silencing response (Chu and Rana, 2008), and (ii) the efficiency of tiny locked nucleic acid (LNA) oligonucleotides as short as $8 \mathrm{nt}$ to antagonize the function of microRNAs harboring a complementary seed region (Obad et al., 2011), respectively.

Generated together with the microRNA that it may regulate, smiRNAs would be a regulator of choice of microRNA function and stability. In fact, the most attractive hypothesis would be that a smiRNA could modulate the function of its microRNA counterpart, i.e., the one encoded by the opposite strand within the same pre-microRNA. In that sense, it may be that smiRNAs can neutralize the function of microRNA species of sufficient base pair complementarity and act as natural microRNA antisense RNAs.

We have previously reported that fragile $\mathrm{X}$ mental retardation protein (FMRP) may facilitate mRNA recognition by microRNAs

\section{REFERENCES}

Bartel, D. P. (2009). MicroRNAs: target recognition and regulatory functions. Cell 136, 215-233.

Boissonneault, V., Plante, I., Rivest, S., and Provost, P. (2009). MicroRNA298 and microRNA-328 regulate expression of mouse beta-amyloid precursor protein-converting enzyme 1. J. Biol. Chem. 284, 1971-1981.

Boissonneault, V., St-Gelais, N., Plante, I., and Provost, P. (2008). A polymerase chain reaction-based cloning strategy applicable to functional microRNA studies. Anal. Biochem. 381, 166-168.

Chendrimada, T. P., Gregory, R. I., Kumaraswamy, E., Norman, J., Cooch, N., Nishikura, K., and Shiekhattar, R. (2005). TRBP recruits the Dicer complex to Ago2 for microRNA processing and gene silencing. Nature 436, 740-744.

Chu, C. Y., and Rana, T. M. (2008). Potent RNAi by short RNA triggers. RNA 14, 1714-1719.

Creighton, C. J., Benham, A. L., Zhu, H., Khan, M. F., Reid, J. G., Nagaraja, A. K., Fountain, M. D., Dziadek, O., Han, D., Ma, L., Kim, J., Hawkins, S. M., Anderson, M. L., Matzuk, M. M., and Gunaratne, P. H. (2010). Discovery of novel microRNAs in female reproductive tract using next generation sequencing. PLoS ONE 5, e9637. doi:10.1371/journal.pone.0009637

Diederichs, S., and Haber, D. A. (2007). Dual role for argonautes in microRNA processing and

through a strand annealing or exchange reactions (Plante et al., 2006). smiRNAs may thus modulate microRNA strand annealing to specific mRNAs or strand exchange between microRNA duplexes and mRNA targets.

Together, these observations led us to propose a model whereby RNA species half the size of microRNAs, tentatively termed semimicroRNAs (or smiRNAs), may be generated along the microRNA pathway and participate to the control of mRNA translation by regulating microRNA activity in vivo (Figure 6). Expanding the family of non-coding RNA species, our study may define smiRNAs as a new class of small regulatory RNAs that may provide an additional layer of control over microRNA activity; the functional significance of smiRNAs, however, remains to be established. The design of therapeutic RNAs aimed to substitute, mimic or inhibit smiRNA function may thus offer a new perspective to the development of novel gene therapeutic strategies.

\section{ACKNOWLEDGMENTS}

Patrick Provost is a Senior Scholar from the Fonds de la Recherche en Santé du Québec (FRSQ). This work was supported by Grant No. 201940 from the Canadian Blood Services/Canadian Institutes of Health Research (CIHR) Blood Utilization and Conservation Initiative.

UTR. Proc. Natl. Acad. Sci. U.S.A 104, 9667-9672.

Ma, J. B., Yuan, Y. R., Meister, G., Pei, Y., Tuschl, T., and Patel, D. J. (2005). Structural basis for $5^{\prime}$-end-specific recognition of guide RNA by the $A$. fulgidus Piwi protein. Nature 434, 666-670.

Macrae, I. J., Zhou, K., Li, F., Repic, A., Brooks, A. N., Cande, W. Z., Adams, P. D., and Doudna, J. A. (2006). Structural basis for double-stranded RNA processing by Dicer. Science 311, 195-198.

Mah, S. M., Buske, C., Humphries, R. K., and Kuchenbauer, F. (2010). miRNA $*$ : a passenger stranded in RNA-induced silencing complex? Crit. Rev. Eukaryot. Gene Expr. 20, 141-148.

Matranga, C., Tomari, Y., Shin, C., Bartel, D. P., and Zamore, P. D. (2005). Passenger-strand cleavage facilitates assembly of siRNA into Ago2-containing RNAi enzyme complexes. Cell 123, 607-620.

Obad, S., Dos Santos, C. O., Petri, A., Heidenblad, M., Broom, O., Ruse, C., Fu, C., Lindow, M., Stenvang, J., Straarup, E. M., Hansen, H. F., Koch, T., Pappin, D., Hannon, G. J., and Kauppinen, S. (2011). Silencing of microRNA families by seedtargeting tiny LNAs. Nat. Genet. 43, 371-378.

Okamura, K., Phillips, M. D., Tyler, D. M., Duan, H., Chou, Y. T., and Lai, E. C. (2008). The regulatory activity of microRNA* species has substantial influence on microRNA and $3^{\prime}$ UTR evolution. Nat. Struct. Mol. Biol. 15, 354-363.

Ouellet, D. L., Perron, M. P., Gobeil, L. A., Plante, P., and Provost, P. (2006). MicroRNAs in gene regulation: when the smallest governs it all. J. Biomed. Biotechnol. 2006, 69616.

Ouellet, D. L., Plante, I., Landry, P., Barat, C., Janelle, M. E., Flamand, L., Tremblay, M. J., and Provost, P. (2008). Identification of functional microRNAs released through asymmetrical processing of HIV-1 TAR element. Nucleic Acids Res. 36, 2353-2365.

Plante, I., Davidovic, L., Ouellet, D. L., Gobeil, L. A., Tremblay, S., Khandjian, E. W., and Provost, P. (2006). Dicer-derived microRNAs are utilized by the fragile $\mathrm{X}$ mental retardation protein for assembly on target RNAs. J. Biomed. Biotechnol. 2006, 64347.

Provost, P., Dishart, D., Doucet, J., Frendewey, D., Samuelsson, B., and Radmark, O. (2002). Ribonuclease activity and RNA binding of recombinant human Dicer. EMBO J. 21, 5864-5874.

Provost, P., Samuelsson, B., and Radmark, O. (1999). Interaction of 5 lipoxygenase with cellular proteins. Proc. Natl. Acad. Sci. U.S.A. 96, 1881-1885.

Qin, W., Shi, Y., Zhao, B., Yao, C., Jin, L., Ma, J., and Jin, Y. (2010). miR24 regulates apoptosis by targeting the open reading frame (ORF) region of FAF1 in cancer cells. PLoS ONE 5, e9429. doi:10.1371/journal.pone.0009429 
Redko, Y., Bechhofer, D. H., and Condon, C. (2008). Mini-III, an unusual member of the RNase III family of enzymes, catalyses $23 \mathrm{~S}$ ribosomal RNA maturation in B. subtilis. Mol. Microbiol. 68, 1096-1106.

Sun, W., Jun, E., and Nicholson, A. W. (2001). Intrinsic doublestranded-RNA processing activity of Escherichia coli ribonuclease III lacking the dsRNA-binding domain. Biochemistry 40, 14976-14984.

Vasudevan, S., Tong, Y., and Steitz, J. A. (2007). Switching from repression to activation: microRNAs can up-regulate translation. Science 318 , 1931-1934.
Wang, Y., Sheng, G., Juranek, S., Tuschl, T., and Patel, D. J. (2008). Structure of the guidestrand-containing argonaute silencing complex. Nature 456, 209-213.

Weitzer, S., and Martinez, J. (2007). The human RNA kinase hClpl is active on $3^{\prime}$ transfer RNA exons and short interfering RNAs. Nature 447, 222-226.

Yang, J. S., Phillips, M. D., Betel, D., Mu, P., Ventura, A., Siepel, A. C., Chen, K. C., and Lai, E. C. (2011). Widespread regulatory activity of vertebrate microRNA* species. RNA 17, 312-326.
Zhang, H., Kolb, F. A., Brondani, V., Billy, E., and Filipowicz, W. (2002). Human Dicer preferentially cleaves dsRNAs at their termini without a requirement for ATP. EMBO J. 21, 5875-5885.

Conflict of Interest Statement: The authors declare that the research was conducted in the absence of any commercial or financial relationships that could be construed as a potential conflict of interest.

Received: 24 January 2012; accepted: 14 May 2012; published online: 04 June 2012.
Citation: Plante I, Plé H, Landry $P$, Gunaratne PH and Provost P (2012) Modulation of microRNA activity by semi-microRNAs. Front. Gene. 3:99. doi: 10.3389/fgene.2012.00099

This article was submitted to Frontiers in Non-Coding RNA, a specialty of Frontiers in Genetics.

Copyright (c) 2012 Plante, Plé, Landry, Gunaratne and Provost. This is an openaccess article distributed under the terms of the Creative Commons Attribution Non Commercial License, which permits non-commercial use, distribution, and reproduction in other forums, provided the original authors and source are credited. 


\section{APPENDIX}

MODULATION OF MICRORNA ACTIVITY BY SEMI-MICRORNAS

Isabelle Plante, Hélène Plé, Patricia Landry, Preethi H. Gunaratne and Patrick Provost

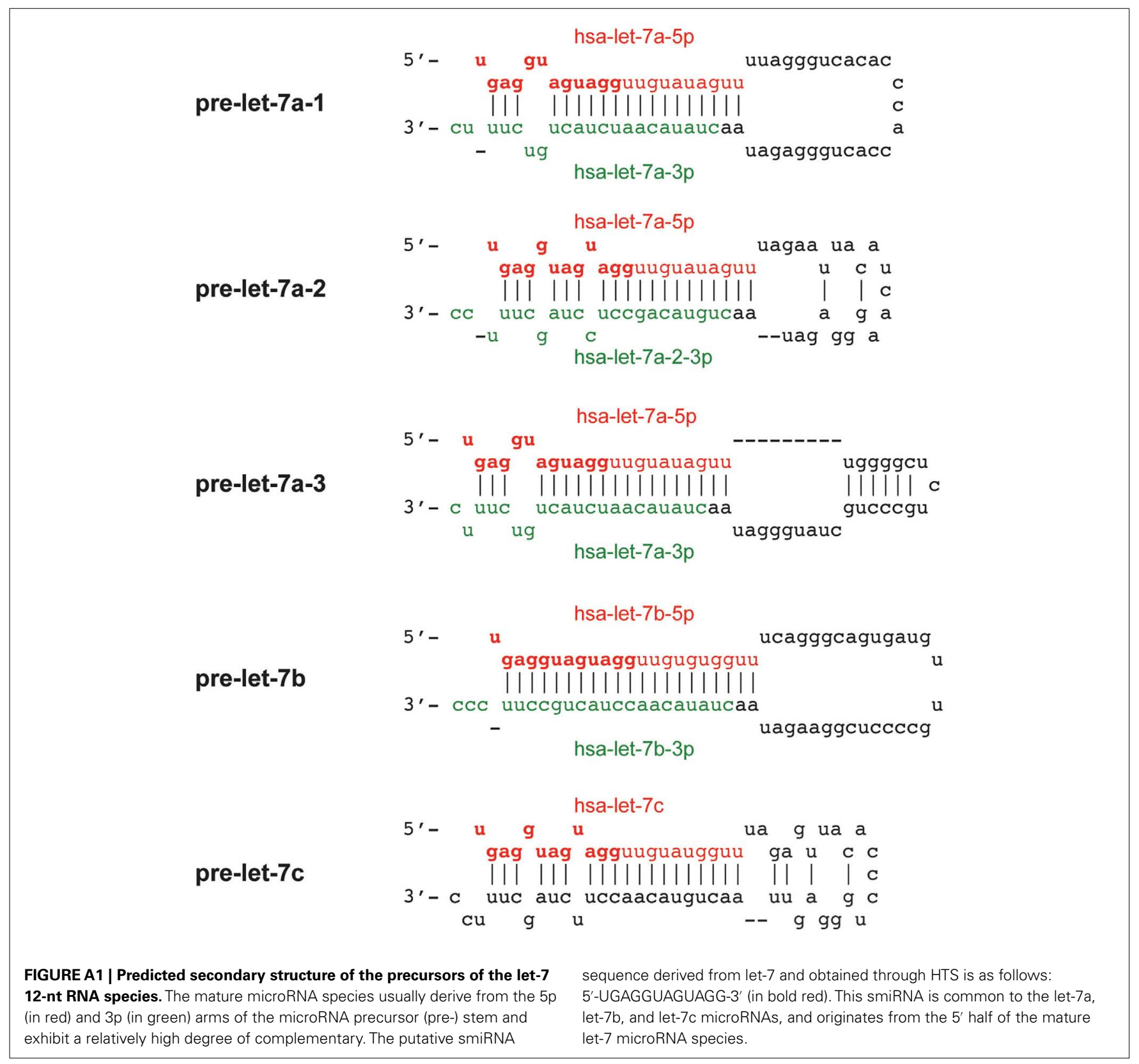

